OPEN ACCESS

Edited by:

Nick J. Davis,

Manchester Metropolitan University,

UK

Reviewed by:

Charlotte K. Häger,

Umeå University, Sweden

Bernadette Ann Murphy,

University of Ontario Institute of

Technology, Canada

*Correspondence:

Javier Márquez-Ruiz

jmarquez@upo.es

Specialty section:

This article was submitted to

Movement Science and Sport

Psychology,

a section of the journal

Frontiers in Psychology

Received: 30 July 2016 Accepted: 05 December 2016 Published: 23 December 2016

Citation:

Ammann $C$, Spampinato $D$ and Márquez-Ruiz J (2016) Modulating Motor Learning through Transcranial Direct-Current Stimulation: An Integrative View.

Front. Psychol. 7:1981. doi: 10.3389/fpsyg.2016.01981

\section{Modulating Motor Learning through Transcranial Direct-Current Stimulation: An Integrative View}

\author{
Claudia Ammann ${ }^{1}$, Danny Spampinato ${ }^{1}$ and Javier Márquez-Ruiz ${ }^{2 *}$ \\ ${ }^{1}$ Department of Physical Medicine and Rehabilitation, Johns Hopkins Medical Institution, Baltimore, MD, USA, ${ }^{2}$ Division of \\ Neurosciences, Pablo de Olavide University, Seville, Spain
}

Motor learning consists of the ability to improve motor actions through practice playing a major role in the acquisition of skills required for high-performance sports or motor function recovery after brain lesions. During the last decades, it has been reported that transcranial direct-current stimulation (tDCS), consisting in applying weak direct current through the scalp, is able of inducing polarity-specific changes in the excitability of cortical neurons. This low-cost, painless and well-tolerated portable technique has found a wide-spread use in the motor learning domain where it has been successfully applied to enhance motor learning in healthy individuals and for motor recovery after brain lesion as well as in pathological states associated to motor deficits. The main objective of this mini-review is to offer an integrative view about the potential use of tDCS for human motor learning modulation. Furthermore, we introduce the basic mechanisms underlying immediate and long-term effects associated to tDCS along with important considerations about its limitations and progression in recent years.

Keywords: transcranial electrical stimulation, tDCS, motor learning, non-invasive brain stimulation, plasticity, skill learning, motor adaptation, use-dependent learning

\section{INTRODUCTION}

Motor learning entails improving motor actions through practice (Willingham, 1998; Dayan and Cohen, 2011; Wolpert et al., 2011). We make use of this ability when acquiring new motor skills and when adapting our movements to account for predictable changes to our environment. Motor learning plays a critical role in acquiring the motor actions necessary for high-performance sports (Nielsen and Cohen, 2008) and for motor recovery after brain lesions (Kitago and Krakauer, 2013). Applying weak direct current through the scalp induces polarity-specific changes in the excitability of cortical neurons (Nitsche et al., 2008; Brunoni et al., 2012). This effect of transcranial directcurrent stimulation (tDCS) was first demonstrated in the human motor cortex (Nitsche and Paulus, 2000, 2001), but has also been described for other brain regions such as visual (Antal et al., 2001, 2004), somatosensory (Rogalewski et al., 2004; Dieckhöfer et al., 2006), prefrontal (Fregni et al., 2005; Mulquiney et al., 2011) and cerebellar cortices (Galea et al., 2009; Grimaldi et al., 2014).

Abbreviations: atDCS, anodal transcranial direct-current stimulation; BDNF, brain-derived neurotrophic factor; ctDCS, cathodal transcranial direct-current stimulation; $\mathrm{GABA}_{\mathrm{A}}, \gamma$-aminobutyric acid type A; HD-tDCS, high-definition transcranial direct-current stimulation; M1, primary motor cortex; MEP, motor evoked potential; NMDA, N-methylD-aspartate; PFC, prefrontal cortex; PM, premotor area; RTs, reaction times; SFTT, serial finger tapping tasks; SMA, supplementary motor area; SRTT, serial reaction time task; SVIPT, sequential visual isometric pinch task; tDCS, transcranial direct-current stimulation; TMS, transcranial magnetic stimulation; UDL, use-dependent learning. 
The modulatory effects and simplicity of tDCS have caught the attention of both basic and clinical neuroresearchers for its potential to modulate motor learning (Lang et al., 2003; Nitsche et al., 2003; Antal et al., 2004; Reis et al., 2008; López-Alonso et al., 2015). Most studies using tDCS deliver a low-current intensity (1-2 $\mathrm{mA}$ ) between two rubber electrodes $\left(25-35 \mathrm{~cm}^{2}\right)$ placed on the scalp for 10-20 min (Stagg and Nitsche, 2011). For this montage, the stimulating electrode is placed over the region of interest while the reference electrode is placed over either the contralateral supraorbital, the mastoid or shoulder. Following this procedure, researchers have utilized tDCS to enhance motor learning in healthy individuals (Reis et al., 2008) and for motor recovery due to brain lesions or pathological states linked to motor deficits (Demirtas-Tatlidede et al., 2012; Grimaldi et al., 2014). tDCS has also been proposed to improve motor capacities and muscle endurance of high-performance sport athletes (Cogiamanian et al., 2007; Banissy and Muggleton, 2013; Williams et al., 2013). Although tDCS application in the motor domain is vast, the main objective of this review is to offer an integrative view of the main findings from studies using cerebral and cerebellar tDCS application in healthy human participants.

\section{BASIC MECHANISMS UNDERLYING tDCS}

Although there is increasing interest for using tDCS as a noninvasive neuromodulation technique, little is known about the molecular and/or cellular mechanisms underlying its effects (Márquez-Ruiz et al., 2012). Since Nitsche and Paulus (2000) described the impact of transcranial low current over the human primary motor cortex (M1), excitatory/inhibitory effects have been broadly associated to anodal/cathodal current stimulation, respectively. However, the net effect of tDCS depends on the stimulated brain region (Dieckhöfer et al., 2006), the number of tDCS sessions (Monte-Silva et al., 2013), the applied current intensity (Batsikadze et al., 2013), and the brain state (Silvanto and Pascual-Leone, 2008; Krause and Cohen Kadosh, 2014) among other parameters. To understand the physiological mechanisms underlying these effects, it is important to disassociate: a) the immediate tDCS effects observed in cells exposed to simultaneous exogenous electrical fields and b) effects mediated by protein modifications requiring longer stimulation periods, lasting for several minutes after tDCS application. The immediate effects are elicited when an external electric field causes displacement of intracellular ions, thus altering the internal charge distribution and modifying the neuronal membrane potential (Ruffini et al., 2013; MárquezRuiz et al., 2014). Moreover, animal studies have shown both neuronal morphology (Radman et al., 2009) and axonal orientation (Kabakov et al., 2012) are critical to consider when explaining tDCS-induced responses, since the maximal effects occur when electric fields are applied parallel to the somatodendritic axis (Bikson et al., 2004). Beyond these somatic changes, animal studies have also demonstrated the importance of presynaptic effects during current application (Kabakov et al., 2012; Márquez-Ruiz et al., 2012; Bikson et al., 2013). The longterm effects, measured indirectly in human studies (recording motor evoked potentials, MEPs, elicited by transcranial magnetic pulses over M1) are mediated by N-methyl-D-aspartate (NMDA) and $\gamma$-aminobutyric acid type $\mathrm{A}\left(\mathrm{GABA}_{\mathrm{A}}\right)$ receptors (see for review Stagg and Nitsche, 2011). Animal studies have confirmed the involvement of NMDA receptors and brainderived neurotrophic factor (BDNF) (Fritsch et al., 2010) for the long-term effects observed after anodal direct-current stimulation (atDCS), and adenosine A1 receptors (Márquez-Ruiz et al., 2012) after cathodal direct-current stimulation (ctDCS).

\section{MODULATING MOTOR LEARNING PROCESSES THROUGH tDCS}

Motor learning encompasses various forms of learning, including, but not exclusive to error-based, reinforcement, use-dependent plasticity, and cognitive strategies (Krakauer and Mazzoni, 2011), each likely involving different neuronal substrates. It becomes more complicated given that these forms of learning likely all contribute to the learning process when acquiring a new skill (Kitago and Krakauer, 2013). Therefore, for better comprehensibility, we grouped publications based on different motor learning paradigms and not the different forms of learning, to explore the impact of tDCS on specific motor behaviors (see Table 1). We included adaptation, skill, and use-dependent repetition (i.e., repeated practice of simple movements) tasks. Undoubtedly, the number of positive findings described below, highlight the potential of tDCS for (1) modulating new behavior acquisition and retention, (2) identifying the underlying learning processes, and (3) studying the role of different brain regions.

\section{Modulating Skill Learning}

Skill learning refers to a process that results in improving the trade-off between speed and accuracy (Reis et al., 2009), typically achieved by reducing movement variability (Smuelof et al., 2012). Investigations have used tDCS to either modulate learning or to better understand the underlying learning processes (Orban de Xivry and Shadmehr, 2014; Savic and Meier, 2016). However, the number of brain regions involved in skill learning is vast (Ungerleider et al., 2002) which has led to various targeted brain regions for tDCS application, electrode montages, and types of motor tasks. The leading paradigms combined with tDCS are motor sequence tasks, including serial reaction time task (SRTT), sequential finger tapping tasks (SFTT), and sequential visual isometric pinch task (SVIPT) (see Table 2 for details).

Several studies have reported enhanced SRTT performance and retention with simultaneously applying atDCS over M1. This is shown by reduced reaction times (RTs), a common way to quantify sequence acquisition (Nitsche et al., 2003; Kang and Paik, 2011; Kantak et al., 2012; Ehsani et al., 2016). Comparably reduced RTs were found during the recall of a sequence task when tDCS was applied over premotor (PM) cortex throughout REM sleep (Nitsche et al., 2010). A few studies, however, have presented null effects of tDCS on RTs, specifically when stimulation was not applied during training 


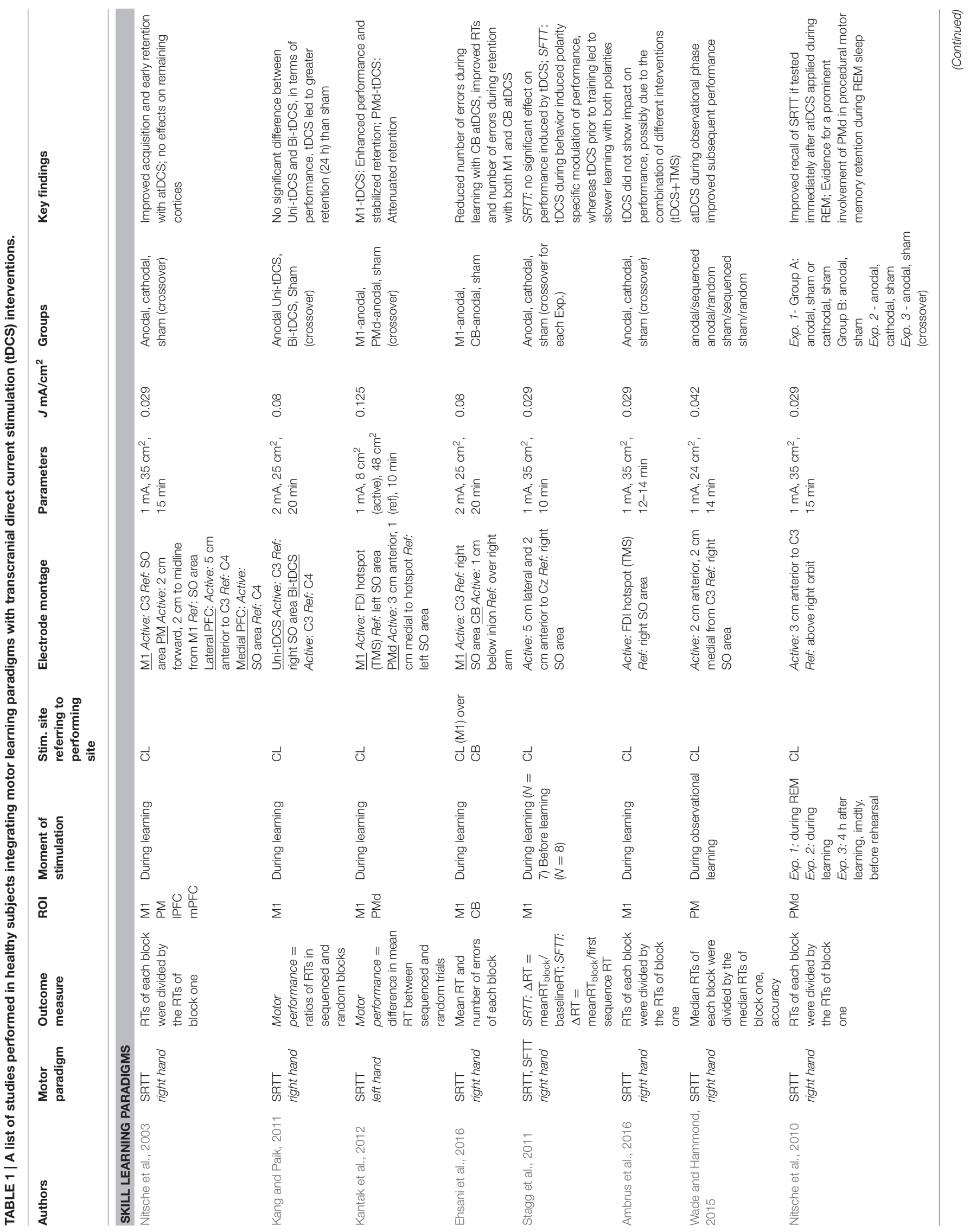




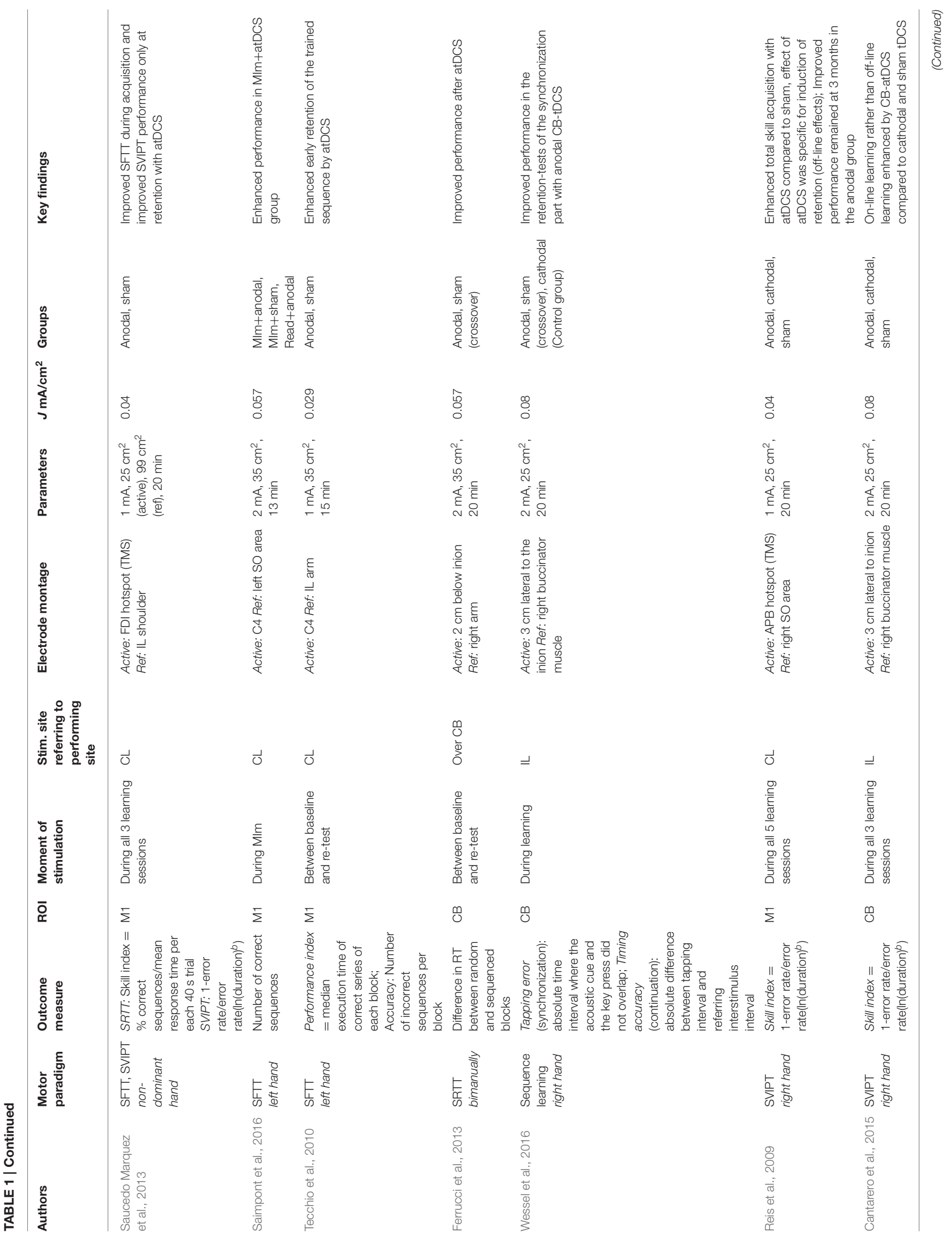




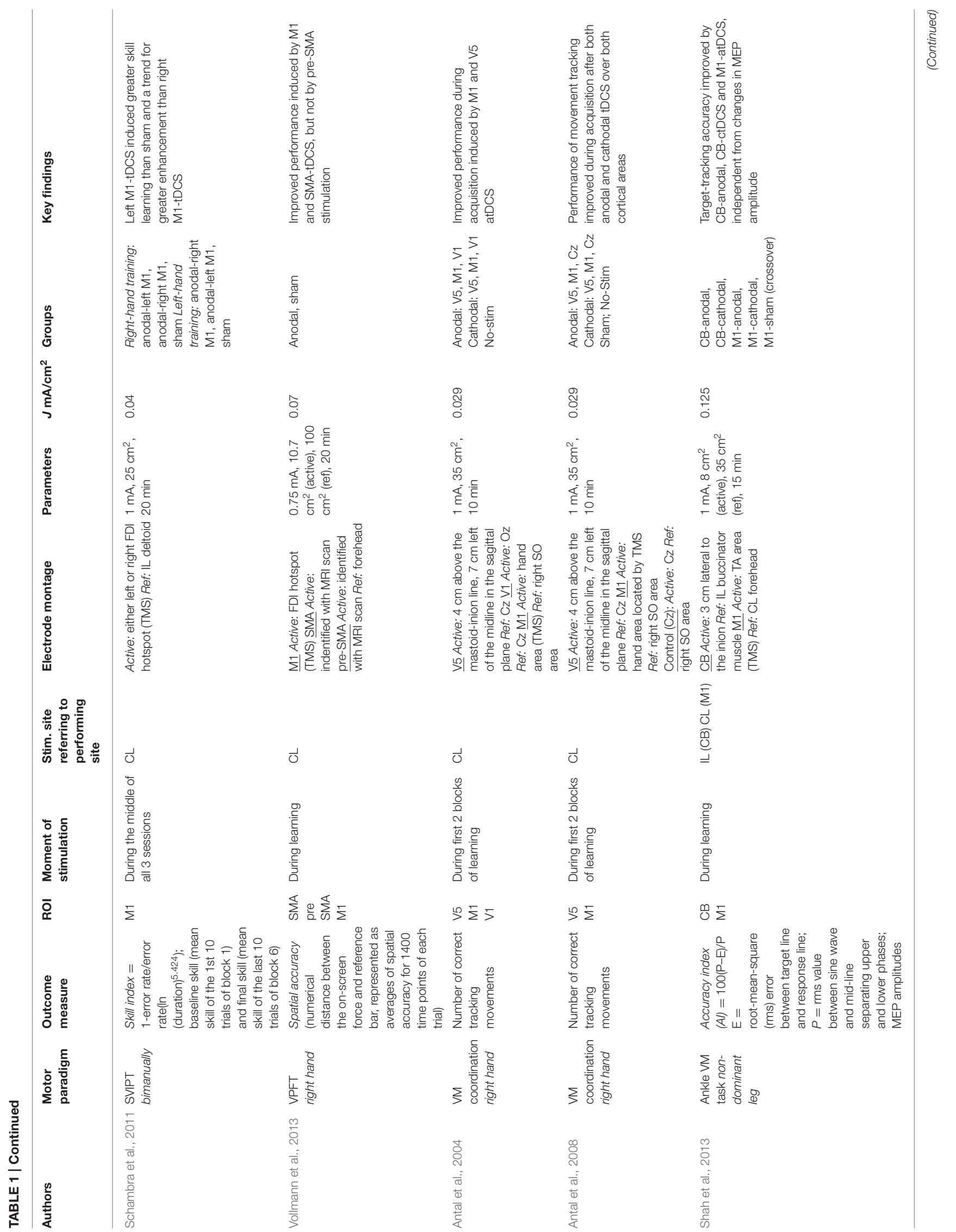




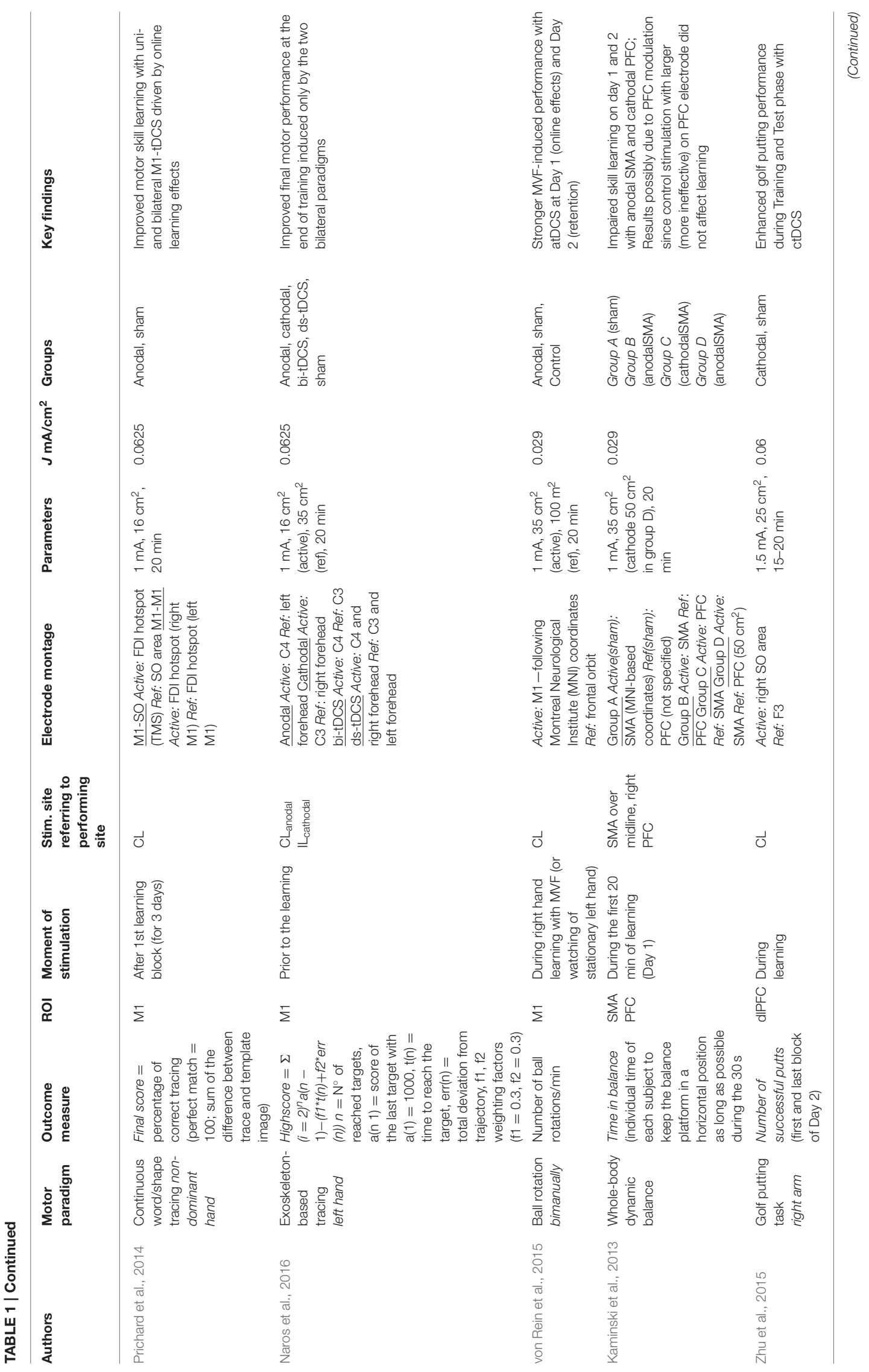




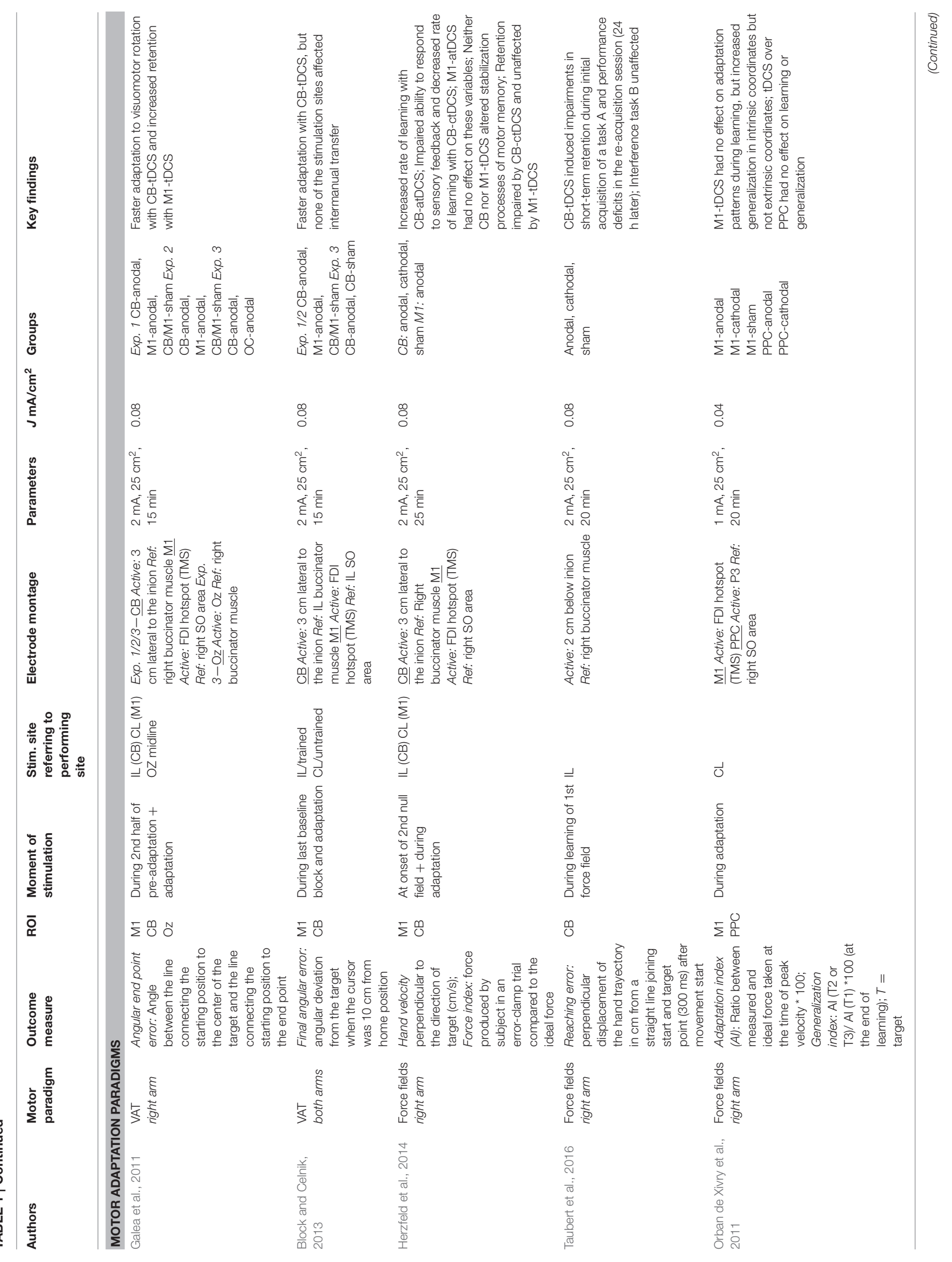




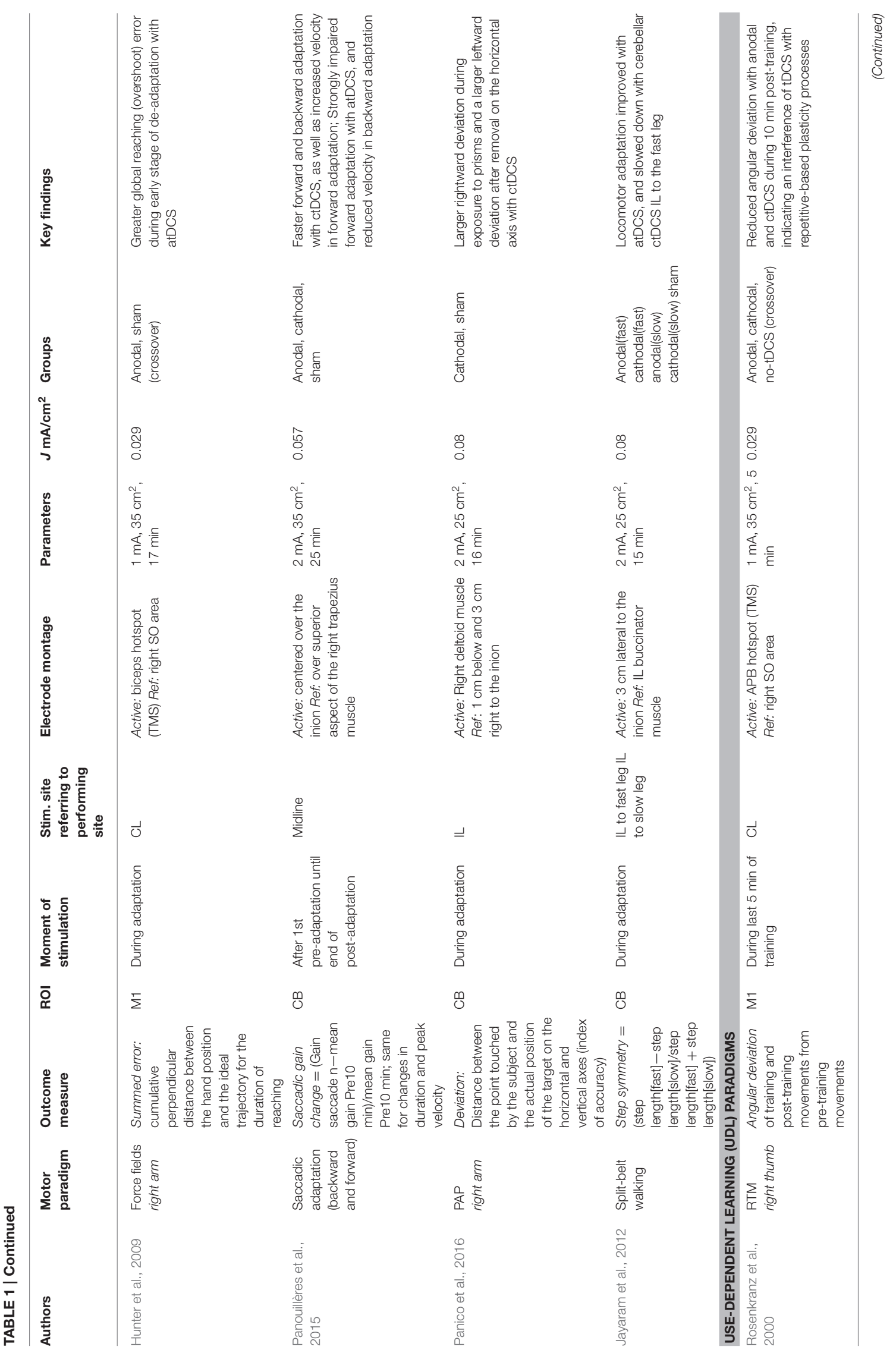




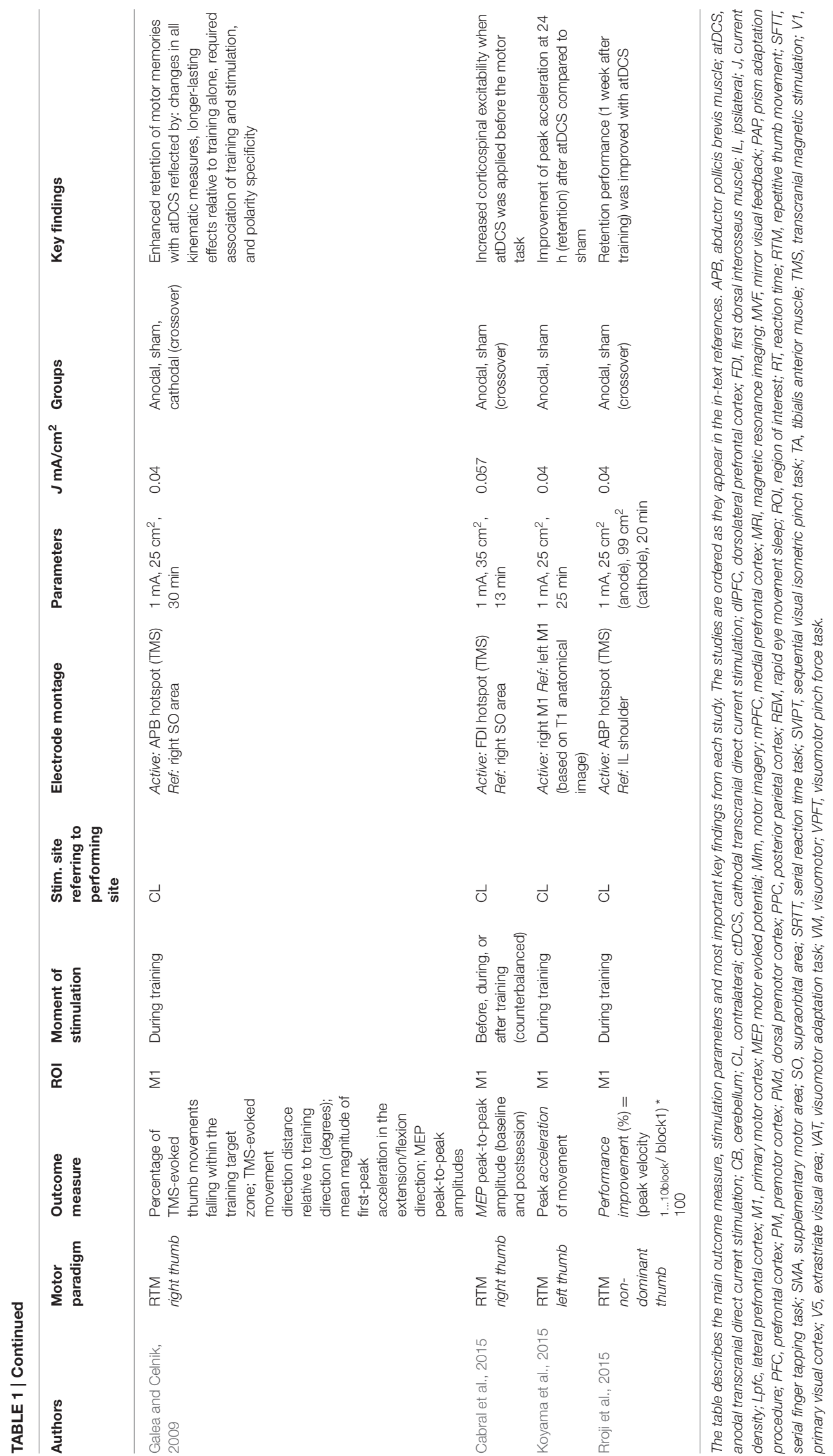


TABLE 2 | Characterization of the main motor paradigms described in this mini-review.

\begin{tabular}{ll}
\hline Motor task & Description \\
\hline SRTT & $\begin{array}{l}\text { Participants respond to visual cues presented on a screen by } \\
\text { pressing an associated keyboard response. The position of the } \\
\text { visual cue is either presented in a repeating sequence or random. }\end{array}$ \\
\hline SFTT & $\begin{array}{l}\text { A specific order of sequence elements is presented on a screen } \\
\text { that present specific finger movements. Participants are instructed } \\
\text { to make the representative key-presses as fast and accurate as } \\
\text { possible. }\end{array}$ \\
\hline SVIPT & $\begin{array}{l}\text { Participants control the movement of a cursor displayed on a } \\
\text { computer screen by squeezing an isometric force transducer } \\
\text { using the thumb and index finger. The aim is to move the cursor as } \\
\text { quickly and accurately as possible between the start position and } \\
\text { a numbered order of target zones. The magnitude of pinch force } \\
\text { applied to the sensor is non-linearly (usually a logarithmic } \\
\text { transduction is applied) related to the displacement of the cursor. }\end{array}$ \\
\hline $\begin{array}{l}\text { Similar to the SVIPT, participants match their own pinch force } \\
\text { visually displayed by a force bar on a computer screen with the } \\
\text { height of a moving reference bar by squeezing a force transducer. }\end{array}$ \\
$\begin{array}{l}\text { Participants make hand-reaching movements with a pen over a } \\
\text { horizontal digitizing tablet to respond to a target displayed on a } \\
\text { vertical screen. Vision of the hand was not visible to participants, } \\
\text { but a cursor on the screen was given to participants to represent } \\
\text { the position of their hand. Participants are instructed to make } \\
\text { rapid and straight uncorrected movements throughout training. } \\
\text { After some practice, a perturbation is introduced by applying a } \\
\text { visual rotation (e.g., by } 30^{\circ} \text { counterclockwise) of the cursor. } \\
\text { Participants adapt incrementally their movements to the new } \\
\text { position and show large and prolonged after-effects once the } \\
\text { perturbation is removed. }\end{array}$ \\
\hline VAT
\end{tabular}

Force fields Participants hold a robotic arm handle in order to make reaching movements to a specific target displayed on a screen. Vision of the hand was obstructed, however, visual feedback of hand position is provided on the screen. After baseline performance, reaching is perturbed by a force field that pushes the hand perpendicular to the direction of movement. After participants adapt to the force field perturbation, participants show large after-effects when the perturbation is removed.

SFTT, sequential finger tapping task; SRTT, serial reaction time task; SVIPT, sequential visual isometric pinch task; VAT, visuomotor adaptation task; VPFT, visual pinch force task.

(Stagg et al., 2011), or when tDCS was combined with singlepulse TMS, causing a potential reduction of tDCS' efficacy (Ambrus et al., 2016). Moreover, when tDCS was applied over PM during SRTT, neither acquisition nor consolidation was modulated (Nitsche et al., 2003), but instead interfered with the retention of learned sequences (Kantak et al., 2012). In contrast, when PM-tDCS was applied while participants watched a video of a hand performing key-press sequences prior to training, RTs were reduced in comparison to sham stimulation. This suggests that increasing excitability of a region involved in action observation promotes skill acquisition (Wade and Hammond, 2015). Additional studies have revealed significant benefits of tDCS on SFTT learning. Interestingly, the number of correctly executed sequences increased both when M1-tDCS was applied concurrently with performance (Saucedo Marquez et al., 2013), and when tDCS was applied during motor imagery of sequences (Saimpont et al., 2016). When individuals received M1-atDCS during performance, RTs decreased during training (Stagg et al., 2011), whereas when M1-atDCS was applied between two training sessions, reduced execution time of correct sequences was found during early consolidation (Tecchio et al., 2010), i.e., stabilization of the motor memory rapidly after its initial acquisition (Brashers-Krug et al., 1996). Together, this suggests M1 as an important site for storage of motor sequences. On the other hand, the role of the cerebellum, a structure critical for motor adaptation (Tseng et al., 2007; Donchin et al., 2012; Izawa et al., 2012), is not well understood for procedural sequence learning (Jenkins et al., 1994; Doyon et al., 2002; Shimizu et al., 2016). Only a few studies have addressed the effects of cerebellar atDCS on sequence learning. For example, cerebellar stimulation applied during SRTT performance reduced the error rate (Ehsani et al., 2016), whereas it reduced RTs when applied prior to a follow-up session (Ferrucci et al., 2013). Interestingly, both M1 and cerebellar atDCS showed enhanced retention of SRTT performance (Ehsani et al., 2016). In a different type of sequence learning which relies on lateral cerebellar function, atDCS over cerebellum reduced tapping movement errors in follow-up sessions. Thus, it appears cerebellar tDCS may facilitate retention of complex motor skills (Wessel et al., 2016).

Simultaneously applying M1-atDCS during SVIPT learning facilitated skill acquisition over several consecutive days of training (Reis et al., 2009; Schambra et al., 2011; Saucedo Marquez et al., 2013). Specifically, stimulation promoted between-session (Reis et al., 2009) or long-term retention processes (Saucedo Marquez et al., 2013). Interestingly, when atDCS was applied over the cerebellum, skill acquisition was enhanced within-session (online) rather than between-session gains. Here, skill improvement was marked by lower errorrates rather than movement time (Cantarero et al., 2015). In a slightly different task (visuo-motor pinch force task, see Table 2 for details), tDCS over secondary motor areas such as the supplementary motor area (SMA) showed to increase participants' spatial accuracy, providing new insights about the role of SMA during skill performance (Vollmann et al., 2013).

Beyond the SRTT, SFTT, and SVIPT tasks, there are additional investigations with varying tasks that have explored tDCS effects during skill learning. For instance, atDCS applied either over M1 or an extrastriate visual area during a visuo-motor coordination task improved early performance of correctly tracked movements (Antal et al., 2004), whereas performance was enhanced for both tDCS polarities when stimulation was applied prior to training (Antal et al., 2008). Moreover, both uni-lateral and bi-lateral M1-tDCS applied concurrently with skill tracing tasks showed enhanced target-tracking accuracy (Shah et al., 2013; Prichard et al., 2014; Naros et al., 2016), an effect similarly found when pairing training with anodal and cathodal cerebellar tDCS (Shah et al., 2013). Furthermore, combining mirror visual feedback with M1-atDCS improved performance of a manual ball-rotation task with the untrained hand, likely due to additive effects on motor performance (von Rein et al., 2015). Accordingly, when the anode electrode was placed over SMA and cathode over right 
prefrontal cortex (PFC) performance of a dynamic whole body task was impaired (Kaminski et al., 2013). On the other side, PFC-ctDCS improved performance of a golf-putting task during acquisition and retention, highlighting a promising application of tDCS toward everyday motor activities (Zhu et al., 2015).

\section{Modulating Motor Adaptation}

Another type of learning studied in laboratory settings is motor adaptation, or a reduction of errors in response to environmental changes via generating an internal model to predict the consequences of actions. Adaptation is generally tested in a variety of error-based tasks (prisms, rotations, force fields), where quickly accounting for perturbations leads to large behavioral changes (Krakauer and Mazzoni, 2011). In relation to brain stimulation, a recent study applied tDCS to distinct brain regions while participants learned a visuomotor rotation (see Table 2 for details). Specifically, they found cerebellar atDCS resulted in faster reduction of errors caused by a consistent visuomotor-rotation (Galea et al., 2011; Block and Celnik, 2013), whereas atDCS over M1 showed a marked increase in retention of the newly learned rotation (Galea et al., 2011). By using tDCS, this study was able to show an important dissociation in acquisition and retention processes related to motor adaptation and further highlighted the distinct roles of the cerebellum and motor cortex. Furthermore, tDCS over these regions did not enhance intermanual transfer of visuomotor rotation learning (Block and Celnik, 2013) suggesting that these structures do not play as critical of a role for this process.

Another study tested tDCS over cerebellum and M1 during force-field adaptation (see Table 2 for details) and consistent with the results reported by Galea et al. (2011), the authors found that cerebellar atDCS enhanced the rate of acquisition (Herzfeld et al., 2014). This study also showed that cerebellar ctDCS delayed the feedback response to the introduced perturbation and decreased the learning rate. Taubert et al. (2016) observed impaired adaptation and re-acquisition of a force-field perturbation with cerebellar atDCS, while no effect was found for ctDCS. It is possible that the experimental design differences of these studies may explain the inconsistent findings.

Regarding the role of M1 in force-field adaptation, M1-tDCS did not alter the rate of adaptation learning during reaching movements (Orban de Xivry et al., 2011; Herzfeld et al., 2014) similar to visuomotor adaptation. While most studies have reported that motor adaptation is not affected by M1-tDCS, one study showed atDCS over M1 biceps brachii representation led to greater overshooting errors in force-field learning once the field was removed, suggesting a possible role of M1 in the adaptation process of reaching movements (Hunter et al., 2009). While these results remain inconclusive, M1-tDCS showed a clear increase of generalization in intrinsic coordinates for joints and muscles during force-field adaptation, without changing extrinsic generalization patterns. In contrast, tDCS tested over posterior parietal cortex had no effects on learning or generalization (Orban de Xivry et al., 2011).

A few studies have also used tDCS to examine functions of the cerebellum outside of visuomotor and force-field adaptation. One study showed that cerebellar excitability plays a crucial role in saccadic adaptation (Panouillères et al., 2015), as well as in all stages of prism adaptation, i.e., in flexible motor adjustments in response to changes of the visual field (Panico et al., 2016). Moreover, Jayaram et al. (2012) were able to modulate locomotor adaptation by applying tDCS over the cerebellum while participants walked on a split-belt treadmill at two different speeds. They found atDCS ipsilateral to the fast leg accelerated adaptation (i.e., promoted faster gait step-symmetry), whereas ctDCS slowed adaptation. Interestingly, atDCS effects primarily affected spatial, rather than temporal components of walking (Jayaram et al., 2012).

\section{Modulating Use-Dependent Learning}

Use-dependent learning (UDL) describes a phenomenon where short-term motor memories are formed and retained due to repeatedly trained motor actions, thus inducing representational changes in the motor cortex (Classen et al., 1998). Rosenkranz et al. (2000) first addressed the effects of tDCS over M1 on UDL by comparing the directional variation of TMS-induced thumb movements (opposite to the trained direction) before and after tDCS application. They found that applying tDCS during the last $5 \mathrm{~min}$ of 30 -min thumb-movement training resulted in smaller TMS-induced angular deviation compared to controls. In other words, anodal or cathodal tDCS with training produced a movement direction similar to the pretraining direction, whereas movements of the control group were biased to the trained direction. The authors concluded that tDCS preserves pre-training cortical movements by interfering with the mechanisms of UDL and the formation of motor memories (Rosenkranz et al., 2000). In contrast, Galea and Celnik (2009) demonstrated enhanced retention effects of repetitive thumb training when atDCS over M1 was applied throughout the $30 \mathrm{~min}$ training period. Importantly, cathodal and sham group responses did not show significant changes. The inconsistencies between these two studies could potentially be explained by the different stimulation periods of tDCS ( 5 vs. $30 \mathrm{~min}$ ). On the other hand, the prior state of the system (i.e., $25 \mathrm{~min}$ of training vs. no training) may not be the same when tDCS is applied at training onset vs. at the end of training (Galea and Celnik, 2009). A recent study aimed to determine whether M1-tDCS applied before, during, or after motor training enhances UDL. The authors found larger MEP amplitudes (first dorsal interosseous muscle) only when atDCS was applied before motor training. This suggests tDCS prior to training benefits optimization of UDL (Cabral et al., 2015). However, these results are inconsistent with other studies. Galea and Celnik (2009) showed a significant effect on training by applying tDCS during the training, an effect that is similarly found with sequence-learning (Stagg et al., 2011). Furthermore, recent results showed enhanced retention of ballistic thumb movements when M1-atDCS was applied during training when evaluating both peak velocities and accelerations of thumb movements (Koyama et al., 2015; Rroji et al., 2015).

\section{CONSIDERATIONS ON MOTOR LEARNING MODULATION AND NEW PERSPECTIVES}

Overall, the results summarized in this review highlight the need for new stimulation paradigms based on more natural 
and individualized stimulation protocols, aiming to optimize the desired stimulation effects. Variability and contradictions between studies need to be considered, however, this is frequently caused by methodological differences (Paulus, 2011; Horvath et al., 2014, 2015). When considering that different brain regions are likely involved in distinct motor learning processes (Shmuelof and Krakauer, 2011; Penhune and Steele, 2012), the simultaneous (or sequential) electrical stimulation of these areas on the proper polarity and intensity could potentially optimize tCS effects. In this regard, bilateral M1 combined with PFC stimulation has been successfully applied (Vines et al., 2008; Mordillo-Mateos et al., 2012; Leite et al., 2013; Naros et al., 2016). However, the characterization of the effects associated to concomitant stimulation of different brain regions is nearly absent in the literature (Kaminski et al., 2013; Minichino et al., 2015) due to the low focality inherent to the technique and the inability from traditional tDCS devices to simultaneously control multiple stimulation electrodes. Indeed, there has been some progression in recent years. Thus, multifocal tDCS devices using several small-size electrodes (Ruffini et al., 2014), High-Definition tDCS (HD-tDCS) scalp montage $(4 \times$ cathode, surrounding a single central anode, Edwards et al., 2013), or concentric electrodes (Bortoletto et al., 2016) provide evidence for more focal tDCS.

\section{REFERENCES}

Ambrus, G. G., Chaieb, L., Stilling, R., Rothkegel, H., Antal, A., and Paulus, W. (2016). Monitoring transcranial direct current stimulation induced changes in cortical excitability during the serial reaction time task. Neurosci. Lett. 616, 98-104. doi: 10.1016/j.neulet.2016.01.039

Antal, A., Begemeier, S., Nitsche, M. A., and Paulus, W. (2008). Prior state of cortical activity influences subsequent practicing of a visuomotor coordination task. Neuropsychologia 46, 3157-3161. doi: 10.1016/j.neuropsychologia.2008.07.007

Antal, A., Nitsche, M. A., Kincses, T. Z., Kruse, W., Hoffmann, K. P., and Paulus, W. (2004). Facilitation of visuo-motor learning by transcranial direct current stimulation of the motor and extrastriate visual areas in humans. Eur. J. Neurosci. 19, 2888-2892. doi: 10.1111/j.1460-9568.2004.03367.x

Antal, A., Nitsche, M. A., and Paulus, W. (2001). External modulation of visual perception in humans. Neuroreport 12, 3553-3555. doi: 10.1097/00001756-200111160-00036

Banissy, M. J., and Muggleton, N. G. (2013). Transcranial direct current stimulation in sports training: potential approaches. Front. Hum. Neurosci. 7:129. doi: 10.3389/fnhum.2013.00129

Batsikadze, G., Moliadze, V., Paulus, W., Kuo, M. F., and Nitsche, M. A. (2013). Partially non-linear stimulation intensity-dependent effects of direct current stimulation on motor cortex excitability in humans. J. Physiol. 591, 1987-2000. doi: 10.1113/jphysiol.2012.249730

Bikson, M., Inoue, M., Akiyama, H., Deans, J. K., Fox, J. E., Miyakawa, H., et al. (2004). Effects of uniform extracellular DC electric fields on excitability in rat hippocampal slices in vitro. J. Physiol. 557, 175-190. doi: 10.1113/jphysiol.2003.055772

Bikson, M., Reato, D., and Rahman, A. (2013). "Cellular and network effects of transcranial direct current stimulation insights from animal models and brain slice," in Transcranial Brain Stimulation, eds. C. Miniussi, W. Paulus, and P. M. Rossini (Boca Raton, FL: CRC Press), 55-91.

Block, H., and Celnik, P. (2013). Stimulating the cerebellum affects visuomotor adaptation but not intermanual transfer of learning. Cerebellum 12, 781-793. doi: 10.1007/s12311-013-0486-7.

Bortoletto, M., Rodella, C., Salvador, R., Miranda, P. C., and Miniussi, C. (2016). Reduced current spread by concentric electrodes in transcranial Electrical Stimulation (tES). Brain Stimul. 9, 525-528. doi: 10.1016/j.brs.2016.03.001
On the other hand, new devices allowing for EEG recording during simultaneous tDCS also present an excellent tool for the development of individualized stimulation protocols based on the observed individual brain activity (Schestatsky et al., 2013).

Although more investigations are needed to provide a better understanding of the effects induced by tDCS, its impact on motor learning and use for exploring neural substrates underlying motor learning have been successfully demonstrated. In other words, the potential of this technique for basic studies and future clinical treatments seems promising. However, ethical considerations using tDCS for high-performance sports are still a matter of discussion (Reardon, 2016).

\section{AUTHOR CONTRIBUTIONS}

CA and JM contributed to the initial draft, CA, DS, and JM edited the text and wrote the final version of the mini-review.

\section{FUNDING}

This work was funded by the Spanish MINECO-FEDER (BFU2014-53820-P).

Brashers-Krug, T., Shadmehr, R., and Bizzi, E. (1996). Consolidation in human motor memory. Nature 382, 252-255

Brunoni, A. R., Nitsche, M. A., Bolognini, N., Bikson, M., Wagner, T., Merabet, L., et al. (2012). Clinical research with transcranial direct current stimulation (tDCS): challenges and future directions. Brain Stimul. 5, 175-195. doi: 10.1016/j.brs.2011.03.002

Cabral, M. E., Baltar, A., Borba, R., Galvao, S., Santos, L., Fregni, F., et al. (2015). Transcranial direct current stimulation: before, during, or after motor training? Neuroreport 26, 618-622. doi: 10.1097/wnr.0000000000000397

Cantarero, G., Spampinato, D., Reis, J., Ajagbe, L., Thompson, T., Kulkarni, K., et al. (2015). Cerebellar direct current stimulation enhances on-line motor skill acquisition through an effect on accuracy. J. Neurosci. 35, 3285-3290. doi: 10.1523/jneurosci.2885-14.2015

Classen, J., Liepert, J., Wise, S. P., Hallett, M., and Cohen, L. G. (1998). Rapid plasticity of human cortical movement representation induced by practice. J. Neurophysiol. 79, 1117-1123.

Cogiamanian, F., Marceglia, S., Ardolino, G., Barbieri, S., and Priori, A. (2007). Improved isometric force endurance after transcranial direct current stimulation over the human motor cortical areas. Eur. J. Neurosci. 26, 242-249. doi: 10.1111/j.1460-9568.2007.05633.x

Dayan, E., and Cohen, L. G. (2011). Neuroplasticity subserving motor skill learning. Neuron 72, 443-454. doi: 10.1016/j.neuron.2011.10.008

Demirtas-Tatlidede, A., Vahabzadeh-Hagh, A. M., Bernabeu, M., Tormos, J. M., and Pascual-Leone, A. (2012). Noninvasive brain stimulation in traumatic brain injury. J. Head Trauma Rehabil. 27, 274-292. doi: 10.1097/HTR.0b013e318217df55

Dieckhöfer, A., Waberski, T. D., Nitsche, M., Paulus, W., Buchner, H., and Gobbelé, R. (2006). Transcranial direct current stimulation applied over the somatosensory cortex - differential effect on low and high frequency SEPs. Clin. Neurophysiol. 117, 2221-2227. doi: 10.1016/j.clinph.2006.07.136

Donchin, O., Rabe, K., Diedrichsen, J., Lally, N., Schoch, B., Gizewski, E. R., et al. (2012). Cerebellar regions involved in adaptation to force field and visuomotor perturbation. J. Neurophysiol. 107, 134-147. doi: 10.1152/jn.000 07.2011

Doyon, J., Song, A. W., Karni, A., Lalonde, F., Adams, M. M., and Ungerleider, L. G. (2002). Experience-dependent changes in cerebellar contributions to motor sequence learning. Proc. Natl. Acad. Sci. U.S.A. 99, 1017-1022. doi: 10.1073/pnas.022615199 
Edwards, D., Cortes, M., Datta, A., Minhas, P., Wassermann, E. M., and Bikson, M. (2013). Physiological and modeling evidence for focal transcranial electrical brain stimulation in humans: a basis for high-definition tDCS. Neuroimage 74, 266-275. doi: 10.1016/j.neuroimage.2013.01.042

Ehsani, F., Bakhtiary, A. H., Jaberzadeh, S., Talimkhani, A., and Hajihasani, A. (2016). Differential effects of primary motor cortex and cerebellar transcranial direct current stimulation on motor learning in healthy individuals: a randomized double-blind sham-controlled study. Neurosci. Res. 112, 10-19. doi: 10.1016/j.neures.2016.06.003

Ferrucci, R., Brunoni, A. R., Parazzini, M., Vergari, M., Rossi, E., Fumagalli, M., et al. (2013). Modulating human procedural learning by cerebellar transcranial direct current stimulation. Cerebellum 12, 485-492. doi: $10.1007 /$ s12311-012-0436-9

Fregni, F., Boggio, P. S., Nitsche, M., Bermpohl, F., Antal, A., Feredoes, E., et al. (2005). Anodal transcranial direct current stimulation of prefrontal cortex enhances working memory. Exp. Brain Res. 166, 23-30. doi: 10.1007/s00221-005-2334-6

Fritsch, B., Reis, J., Martinowich, K., Schambra, H. M., Ji, Y., Cohen, L. G., et al. (2010). Direct current stimulation promotes BDNF-dependent synaptic plasticity: potential implications for motor learning. Neuron 66, 198-204. doi: 10.1016/j.neuron.2010.03.035

Galea, J. M., and Celnik, P. (2009). Brain polarization enhances the formation and retention of motor memories. J. Neurophysiol. 102, 294-301. doi: 10.1152/jn.00184.2009

Galea, J. M., Jayaram, G., Ajagbe, L., and Celnik, P. (2009). Modulation of cerebellar excitability by polarity-specific noninvasive direct current stimulation. J. Neurosci. 29, 9115-9122. doi: 10.1523/JNEUROSCI.2184-09.2009

Galea, J. M., Vazquez, A., Pasricha, N., de Xivry, J. J., and Celnik, P. (2011). Dissociating the roles of the cerebellum and motor cortex during adaptive learning: the motor cortex retains what the cerebellum learns. Cereb. Cortex 21, 1761-1770. doi: 10.1093/cercor/bhq246

Grimaldi, G., Argyropoulos, G. P., Bastian, A., Cortes, M., Davis, N. J., Edwards, D. J., et al. (2014). Cerebellar transcranial Direct Current Stimulation (ctDCS): a novel approach to understanding cerebellar function in health and disease. Neuroscientist 22, 83-97. doi: 10.1177/1073858414559409

Herzfeld, D. J., Pastor, D., Haith, A. M., Rossetti, Y., Shadmehr, R., and O'Shea, J. (2014). Contributions of the cerebellum and the motor cortex to acquisition and retention of motor memories. Neuroimage 98, 147-158. doi: 10.1016/j.neuroimage.2014.04.076

Horvath, J. C., Carter, O., and Forte, J. D. (2014). Transcranial direct current stimulation: five important issues we aren't discussing (but probably should be). Front. Syst. Neurosci. 8:2. doi: 10.3389/fnsys.2014.00002

Horvath, J. C., Forte, J. D., and Carter, O. (2015). Evidence that transcranial direct current stimulation (tDCS) generates little-to-no reliable neurophysiologic effect beyond MEP amplitude modulation in healthy human subjects: a systematic review. Neuropsychologia 66, 213-236. doi: 10.1016/j.neuropsychologia.2014.11.021

Hunter, T., Sacco, P., Nitsche, M. A., and Turner, D. L. (2009). Modulation of internal model formation during force field-induced motor learning by anodal transcranial direct current stimulation of primary motor cortex. J. Physiol. 587, 2949-2961. doi: 10.1113/jphysiol.2009.169284

Izawa, J., Criscimagna-Hemminger, S. E., and Shadmehr, R. (2012). Cerebellar contributions to reach adaptation and learning sensory consequences of action. J. Neurosci. 32, 4230-4239. doi: 10.1523/jneurosci.6353-11.2012

Jayaram, G., Tang, B., Pallegadda, R., Vasudevan, E. V., Celnik, P., and Bastian, A. (2012). Modulating locomotor adaptation with cerebellar stimulation. J. Neurophysiol. 107, 2950-2957. doi: 10.1152/jn.00645.2011

Jenkins, I. H., Brooks, D. J., Nixon, P. D., Frackowiak, R. S. J., and Passingham, R. E. (1994). Motor sequence learning: a study with positron emission tomography. J Neurosci. 14, 3775-3790.

Kabakov, A. Y., Muller, P. A., Pascual-Leone, A., Jensen, F. E., and Rotenberg, A. (2012). Contribution of axonal orientation to pathway-dependent modulation of excitatory transmission by direct current stimulation in isolated rat hippocampus. J. Neurophysiol. 107, 1881-1889. doi: 10.1152/jn.00715.2011

Kaminski, E., Hoff, M., Sehm, B., Taubert, M., Conde, V., Steele, C. J., et al. (2013). Effect of transcranial direct current stimulation (tDCS) during complex whole body motor skill learning. Neurosci. Lett. 552, 76-80. doi: 10.1016/j.neulet.2013.07.034
Kang, E. K., and Paik, N. J. (2011). Effect of a tDCS electrode montage on implicit motor sequence learning in healthy subjects. Exp. Transl. Stroke. Med. 3:4. doi: 10.1186/2040-7378-3-4

Kantak, S. S., Mummidisetty, C. K., and Stinear, J. W. (2012). Primary motor and premotor cortex in implicit sequence learning-evidence for competition between implicit and explicit human motor memory systems. Eur. J. Neurosci. 36, 2710-2715. doi: 10.1111/j.1460-9568.2012.08175.x

Kitago, T., and Krakauer, J. W. (2013). Motor learning principles for neurorehabilitation. Handb. Clin. Neurol. 110, 93-103. doi: 10.1016/B978-0-444-52901-5.00008-3

Koyama, S., Tanaka, S., Tanabe, S., and Sadato, N. (2015). Dual-hemisphere transcranial direct current stimulation over primary motor cortex enhances consolidation of a ballistic thumb movement. Neurosci. Lett. 588, 49-53. doi: 10.1016/j.neulet.2014.11.043

Krakauer, J. W., and Mazzoni, P. (2011). Human sensorimotor learning: adaptation, skill, and beyond. Curr. Opin. Neurobiol. 21, 636-644. doi: 10.1016/j.conb.2011.06.012

Krause, B., and Cohen Kadosh, R. (2014). Not all brains are created equal: the relevance of individual differences in responsiveness to transcranial electrical stimulation. Front. Syst. Neurosci. 8:25. doi: 10.3389/fnsys.2014.00025

Lang, N., Nitsche, M. A., Sommer, M., Tergau, F., and Paulus, W. (2003). Modulation of motor consolidation by external DC stimulation. Suppl. Clin. Neurophysiol. 56, 277-281. doi: 10.1016/S1567-424X(09)70231-4

Leite, J., Carvalho, S., Fregni, F., Boggio, P. S., and Gonçalves, O. F. (2013). The effects of cross-hemispheric dorsolateral prefrontal cortex transcranial direct current stimulation (tDCS) on task switching. Brain Stimul. 6, 660-667. doi: 10.1016/j.brs.2012.10.006

López-Alonso, V., Cheeran, B., and Fernández-del-Olmo, M. (2015). Relationship between non-invasive brain stimulation-induced plasticity and capacity for motor learning. Brain Stimul. 8, 1209-1219. doi: 10.1016/j.brs.2015.07.042

Márquez-Ruiz, J., Leal-Campanario, R., Sánchez-Campusano, R., MolaeeArdekani, B., Wendling, F., Miranda, P. C., et al. (2012). Transcranial directcurrent stimulation modulates synaptic mechanisms involved in associative learning in behaving rabbits. Proc. Natl. Acad. Sci. U.S.A. 109, 6710-6715. doi: $10.1073 /$ pnas. 1121147109

Márquez-Ruiz, J., Leal-Campanario, R., Wendling, F., Ruffini, G., Gruart, A., and Delgado-García, J. M. (2014). “Transcranial electrical-current stimulation in animals," in The Stimulated Brain: Cognitive Enhancement Using Non-Invasive Brain Stimulation, ed R. Cohen Kadosh (Amsterdam: Elsevier), 117-143. doi: 10.1016/B978-0-12-404704-4.00005-3

Minichino, A., Bersani, F. S., Bernabei, L., Spagnoli, F., Vergnani, L., Corrado, A., et al. (2015). Prefronto-cerebellar transcranial direct current stimulation improves visuospatial memory, executive functions, and neurological soft signs in patients with euthymic bipolar disorder. Neuropsychiatr. Dis. Treat. 11, 2265-2270. doi: 10.2147/NDT.S79108

Monte-Silva, K., Kuo, M. F., Hessenthaler, S., Fresnoza, S., Liebetanz, D., Paulus, W., et al. (2013). Induction of late LTP-like plasticity in the human motor cortex by repeated non-invasive brain stimulation. Brain Stimul. 6, 424-432. doi: 10.1016/j.brs.2012.04.011

Mordillo-Mateos, L., Turpin-Fenoll, L., Millán-Pascual, J., Núñez-Pérez, N., Panyavin, I., Gómez-Argüelles, J. M., et al. (2012). Effects of simultaneous bilateral tDCS of the human motor cortex. Brain Stimul. 5, 214-222. doi: 10.1016/j.brs.2011.05.001

Mulquiney, P. G., Hoy, K. E., Daskalakis, Z. J., and Fitzgerald, P. B. (2011). Improving working memory: exploring the effect of transcranial random noise stimulation and transcranial direct current stimulation on the dorsolateral prefrontal cortex. Clin. Neurophysiol. 122, 2384-2389. doi: 10.1016/j.clinph.2011.05.009

Naros, G., Geyer, M., Koch, S., Mayr, L., Ellinger, T., Grimm, F., et al. (2016). Enhanced motor learning with bilateral transcranial direct current stimulation: impact of polarity or current flow direction? Clin. Neurophysiol. 127, 2119-2126. doi: 10.1016/j.clinph.2015.12.020

Nielsen, J. B., and Cohen, L. G. (2008). The Olympic brain. Does corticospinal plasticity play a role in acquisition of skills required for high-performance sports? J. Physiol. 586, 65-70. doi: 10.1113/jphysiol.2007.142661

Nitsche, M. A., Cohen, L. G., Wassermann, E. M., Priori, A., Lang, N., Antal, A., et al. (2008). Transcranial direct current stimulation: state of the art 2008. Brain Stimul. 1, 206-223. doi: 10.1016/j.brs.2008.06.004 
Nitsche, M. A., Jakoubkova, M., Thirugnanasambandam, N., Schmalfuss, L., Hullemann, S., Sonka, K., et al. (2010). Contribution of the premotor cortex to consolidation of motor sequence learning in humans during sleep. J. Neurophysiol. 104, 2603-2614. doi: 10.1152/jn.00611.2010

Nitsche, M. A., and Paulus, W. (2000). Excitability changes induced in the human motor cortex by weak transcranial direct current stimulation. J. Physiol. 527, 633-639. doi: 10.1111/j.1469-7793.2000.t01-1-00633.x

Nitsche, M. A., and Paulus, W. (2001). Sustained excitability elevations induced by transcranial DC motor cortex stimulation in humans. Neurology 57, 1899-1901. doi: 10.1212/WNL.57.10.1899

Nitsche, M. A., Schauenburg, A., Lang, N., Liebetanz, D., Exner, C., Paulus, W., et al. (2003). Facilitation of implicit motor learning by weak transcranial direct current stimulation of the primary motor cortex in the human. J. Cogn. Neurosci. 15, 619-626. doi: 10.1162/089892903321662994

Orban de Xivry, J. J., Marko, M. K., Pekny, S. E., Pastor, D., Izawa, J., Celnik, P., et al. (2011). Stimulation of the human motor cortex alters generalization patterns of motor learning. J. Neurosci. 31, 7102-7110. doi: 10.1523/jneurosci.0273-11.2011

Orban de Xivry, J. J., and Shadmehr, R. (2014). Electrifying the motor engram: effects of tDCS on motor learning and control. Exp. Brain Res. 232, 3379-3395. doi: 10.1007/s00221-014-4087-6

Panico, F., Sagliano, L., Grossi, D., and Trojano, L. (2016). Cerebellar cathodal tDCS interferes with recalibration and spatial realignment during prism adaptation procedure in healthy subjects. Brain. Cogn. 105, 1-8. doi: 10.1016/j.bandc.2016.03.002

Panouillères, M. T., Miall, R. C., and Jenkinson, N. (2015). The role of the posterior cerebellum in saccadic adaptation: a transcranial direct current stimulation study. J. Neurosci. 35, 5471-5479. doi: 10.1523/jneurosci.4064-14.2015

Paulus, W. (2011). Transcranial electrical stimulation (tES - tDCS; tRNS, tACS) methods. Neuropsychol. Rehabil. 21, 602-617. doi: 10.1080/09602011.2011.557292

Penhune, V. B., and Steele, C. J. (2012). Parallel contributions of cerebellar, striatal and M1 mechanisms to motor sequence learning. Behav. Brain Res. 226, 579-591. doi: 10.1016/j.bbr.2011.09.044

Prichard, G., Weiller, C., Fritsch, B., and Reis, J. (2014). Effects of different electrical brain stimulation protocols on subcomponents of motor skill learning. Brain Stimul. 7, 532-540. doi: 10.1016/j.brs.2014.04.005

Radman, T., Ramos, R. L., Brumberg, J. C., and Bikson, M. (2009). Role of cortical cell type and morphology in subthreshold and suprathreshold uniform electric field stimulation in vitro. Brain Stimul. 2, 215-228. doi: 10.1016/j.brs.2009.03.007

Reardon, S. (2016). 'Brain doping' may improve athletes' performance. Nature 531, 283-284. doi: 10.1038/nature.2016.19534

Reis, J., Robertson, E., Krakauer, J. W., Rothwell, J., Marshall, L., Gerloff, C., et al. (2008). Consensus: "Can tDCS and TMS enhance motor learning and memory formation?" Brain Stimul. 1, 363-369. doi: 10.1016/j.brs.2008.08.001

Reis, J., Schambra, H. M., Cohen, L. G., Buch, E. R., Fritsch, B., Zarahn, E., et al. (2009). Noninvasive cortical stimulation enhances motor skill acquisition over multiple days through an effect on consolidation. Proc. Natl. Acad. Sci. U.S.A. 106, 1590-1595. doi: 10.1073/pnas.0805413106

Rogalewski, A., Breitenstein, C., Nitsche, M. A., Paulus, W., and Knecht, S. (2004). Transcranial direct current stimulation disrupts tactile perception. Eur. J. Neurosci. 20, 313-316. doi: 10.1111/j.0953-816X.2004.03450.x

Rosenkranz, K., Nitsche, M. A., Tergau, F., and Paulus, W. (2000). Diminution of training-induced transient motor cortex plasticity by weak transcranial direct current stimulation in the human. Neurosci. Lett. 296, 61-63. doi: 10.1016/S0304-3940(00)01621-9

Rroji, O., van Kuyck, K., Nuttin, B., and Wenderoth, N. (2015). Anodal tDCS over the primary motor cortex facilitates long-term memory formation reflecting use-dependent plasticity. PLoS ONE 10:e127270. doi: 10.1371/journal.pone.0127270

Ruffini, G., Fox, M. D., Ripolles, O., Miranda, P. C., and Pascual-Leone, A. (2014). Optimization of multifocal transcranial current stimulation for weighted cortical pattern targeting from realistic modeling of electric fields. Neuroimage 89, 216-225. doi: 10.1016/j.neuroimage.2013.12.002

Ruffini, G., Wendling, F., Merlet, I., Molaee-Ardekani, B., Mekonnen, A., Salvador, R., et al. (2013). Transcranial current brain stimulation (tCS): models and technologies. IEEE Trans. Neural. Syst. Rehabil. Eng. 21, 333-345. doi: 10.1109/TNSRE.2012.2200046

Saimpont, A., Mercier, C., Malouin, F., Guillot, A., Collet, C., Doyon, J., et al. (2016). Anodal transcranial direct current stimulation enhances the effects of motor imagery training in a finger tapping task. Eur. J. Neurosci. 43, 113-119. doi: 10.1111/ejn.13122

Saucedo Marquez, C. M., Zhang, X., Swinnen, S. P., Meesen, R., and Wenderoth, N. (2013). Task-specific effect of transcranial direct current stimulation on motor learning. Front. Hum. Neurosci. 7:333. doi: 10.3389/fnhum.2013.00333

Savic, B., and Meier, B. (2016). How transcranial direct current stimulation can modulate implicit motor sequence learning and consolidation: a brief review. Front. Hum. Neurosci. 10:26. doi: 10.3389/fnhum.2016.00026

Schambra, H. M., Abe, M., Luckenbaugh, D. A., Reis, J., Krakauer, J. W., and Cohen, L. G. (2011). Probing for hemispheric specialization for motor skill learning: a transcranial direct current stimulation study. J. Neurophysiol. 106, 652-661. doi: 10.1152/jn.00210.2011

Schestatsky, P., Morales-Quezada, L., and Fregni, F. (2013). Simultaneous EEG monitoring during transcranial direct current stimulation. J. Vis. Exp. 76:50426. doi: $10.3791 / 50426$

Shah, B., Nguyen, T. T., and Madhavan, S. (2013). Polarity independent effects of cerebellar tDCS on short term ankle visuomotor learning. Brain Stimul. 6, 966-968. doi: 10.1016/j.brs.2013.04.008

Shimizu, R. E., Wu, A. D., and Knowlton, B. J. (2016). Cerebellar activation during motor sequence learning is associated with subsequent transfer to new sequences. Behav. Neurosci. 130, 572-584. doi: 10.1037/bne0000146

Shmuelof, L., and Krakauer, J. W. (2011). Are we ready for a natural history of motor learning? Neuron 72, 469-476. doi: 10.1016/j.neuron.2011.10.017

Silvanto, J., and Pascual-Leone, A. (2008). State-dependency of transcranial magnetic stimulation. Brain Topogr. 21, 1-10. doi: 10.1007/s10548-008-0067-0

Smuelof, L., Krakauer, J. W., and Mazzoni, P. (2012). How is a motor skill learned? Change and invariance at the levels of task success and trajectory control. $J$. Neurophysiol. 108, 578-594. doi: 10.1152/jn.00856.2011

Stagg, C. J., Jayaram, G., Pastor, D., Kincses, Z. T., Matthews, P. M., and JohansenBerg, H. (2011). Polarity and timing-dependent effects of transcranial direct current stimulation in explicit motor learning. Neuropsychologia 49, 800-804. doi: 10.1016/j.neuropsychologia.2011.02.009

Stagg, C. J., and Nitsche, M. A. (2011). Physiological basis of transcranial direct current stimulation. Neuroscientist 17, 37-53. doi: 10.1177/10738584103 86614

Taubert, M., Stein, T., Kreutzberg, T., Stockinger, C., Hecker, L., Focke, A., et al. (2016). Remote effects of non-invasive cerebellar stimulation on error processing in motor re-learning. Brain Stimul. 9, 692-699. doi: 10.1016/j.brs.2016.04.007

Tecchio, F., Zappasodi, F., Assenza, G., Tombini, M., Vollaro, S., Barbati, G., et al. (2010). Anodal transcranial direct current stimulation enhances procedural consolidation. J. Neurophysiol. 104, 1134-1140. doi: 10.1152/jn.00661.2009

Tseng, Y. W., Diedrichsen, J., Krakauer, J. W., Shadmehr, R., and Bastian, A. J. (2007). Sensory prediction errors drive cerebellum-dependent adaptation of reaching. J. Neurophysiol. 98, 54-62. doi: 10.1152/jn.00266.2007

Ungerleider, L. G., Doyon, J., and Karni, A. (2002). Imaging brain plasticity during motor skill learning. Neurobiol. Mem. 78, 553-564. doi: 10.1006/nlme.2002.4091

Vines, B. W., Cerruti, C., and Schlaug, G. (2008). Dual-hemisphere tDCS facilitates greater improvements for healthy subjects' non-dominant hand compared to uni-hemisphere stimulation. BMC Neurosci. 9:103. doi: 10.1186/1471-2202-9-103

Vollmann, H., Conde, V., Sewerin, S., Taubert, M., Sehm, B., Witte, O. W., et al. (2013). Anodal transcranial direct current stimulation (tDCS) over supplementary motor area (SMA) but not pre-SMA promotes short-term visuomotor learning. Brain Stimul. 6, 101-107. doi: 10.1016/j.brs.2012.03.018

von Rein, E., Hoff, M., Kaminski, E., Sehm, B., Steele, C. J., Villringer, A., et al. (2015). Improving motor performance without training: the effect of combining mirror visual feedback with transcranial direct current stimulation. J. Neurophysiol. 113, 2383-2389. doi: 10.1152/jn.00832.2014

Wade, S., and Hammond, G. (2015). Anodal transcranial direct current stimulation over premotor cortex facilitates observational learning of a motor sequence. Eur. J. Neurosci. 41, 1597-1602. doi: 10.1111/ejn.12916 
Wessel, M. J., Zimerman, M., Timmermann, J. E., Heise, K. F., Gerloff, C., and Hummel, F. C. (2016). Enhancing consolidation of a new temporal motor skill by cerebellar noninvasive stimulation. Cereb. Cortex 26, 1660-1667. doi: 10.1093/cercor/bhu335

Williams, P. S., Hoffman, R. L., and Clark, B. C. (2013). Preliminary evidence that anodal transcranial direct current stimulation enhances time to task failure of a sustained submaximal contraction. PLOS ONE 8:e81418. doi: 10.1371/journal.pone.0081418

Willingham, D. B. (1998). A neuropsychological theory of motor skill learning. Psychol. Rev. 105, 558-584. doi: 10.1037/0033-295X.105.3.558

Wolpert, D. M., Diedrichsen, J., and Flanagan, J. R. (2011). Principles of sensorimotor learning. Nat. Rev. Neurosci. 12, 739-751. doi: 10.1038/nrn3112

Zhu, F. F., Yeung, A. Y., Poolton, J. M., Lee, T. M., Leung, G. K., and Masters, R. S. (2015). Cathodal transcranial direct current stimulation over left dorsolateral prefrontal cortex area promotes implicit motor learning in a golf putting task. Brain Stimul. 8, 784-786. doi: 10.1016/j.brs.2015.02.005

Conflict of Interest Statement: The authors declare that the research was conducted in the absence of any commercial or financial relationships that could be construed as a potential conflict of interest.

Copyright () 2016 Ammann, Spampinato and Márquez-Ruiz. This is an openaccess article distributed under the terms of the Creative Commons Attribution License (CC BY). The use, distribution or reproduction in other forums is permitted, provided the original author(s) or licensor are credited and that the original publication in this journal is cited, in accordance with accepted academic practice. No use, distribution or reproduction is permitted which does not comply with these terms. 